\title{
Gender differences in clinical, immunological, and virological outcomes in highly active antiretroviral-treated HIV-HCV coinfected patients
}

This article was published in the following Dove Press journal:

Patient Preference and Adherence

4 May 2010

Number of times this article has been viewed

\author{
Joel Emery' \\ Neora Pick ${ }^{2}$ \\ Edward J Mills ${ }^{3}$ \\ Curtis L Cooper' \\ 'The Ottawa Hospital Division \\ of Infectious Diseases, University \\ of Ottawa, Ottawa, Canada; \\ 'Oak Tree Clinic, BC Women's \\ Hospital, Vancouver, Canada; ${ }^{3}$ Faculty \\ of Health Sciences, University of \\ Ottawa, Ottawa, Canada
}

Objective: The influence of biological sex on human immunodeficiency virus (HIV) antiretroviral treatment outcome is not well described in $\mathrm{HIV}$-hepatitis $\mathrm{C}(\mathrm{HCV})$ coinfection.

Methods: We assessed patients' clinical outcomes of HIV-HCV coinfected patients initiating antiretroviral therapy attending the Ottawa Hospital Immunodeficiency Clinic from January 1996 to June 2008.

Results: We assessed 144 males and 39 females. Although similar in most baseline characteristics, the CD4 count was higher in females ( 375 vs 290 cells $/ \mu \mathrm{L}$ ). Fewer females initiated ritonavir-boosted regimens. The median duration on therapy before interruption or change was longer in males (10 versus 4 months) (odds ratio [OR] 1.40 95\% confidence interval: 0.95-2.04; $P=0.09$ ). HIV RNA suppression was frequent (74\%) and mean CD4 count achieved robust (over 400 cells $/ \mu \mathrm{L}$ ) at 6 months, irrespective of sex. The primary reasons for therapy interruption in females and males included: gastrointestinal intolerance ( $25 \%$ vs $19 \% ; P=0.42)$; poor adherence ( $22 \%$ vs $15 \% ; P=0.31$ ); neuropsychiatric symptoms ( $19 \%$ vs $5 \% ; P=0.003$ ); and lost to follow-up ( $3 \%$ vs $13 \% ; P=0.08)$. Seven males $(5 \%)$ and no females discontinued therapy for liver-specific complications. Death rate was higher in females $(23 \%$ vs $7 \% ; P=0.003)$.

Conclusion: There are subtle differences in the characteristics of female and male HIV-HCV coinfected patients that influence HIV treatment decisions. The reasons for treatment interruption and change differ by biological sex. This knowledge should be considered when starting HIV therapy and in efforts to improve treatment outcomes.

Keywords: AIDS, HIV, HCV, coinfection, HAART, viral load, women, gender differences, outcome, CD4 cell counts

\section{Introduction}

The natural history and clinical outcomes in human immunodeficiency virus (HIV) may differ by biological sex. ${ }^{1}$ When highly-active antiretroviral therapy (HAART) first became available, females were less likely to be on therapy and were observed to have diminished therapeutic outcomes..$^{2-4}$ Although more recent data suggest that these gaps have diminished in magnitude, concerns related to decreased access and quality of care for females persist. ${ }^{2,4}$ Although data are conflicting, several studies suggest that women have lower plasma HIV RNA levels than men. ${ }^{1,5}$ Since this measure influences the timing of HAART initiate, it is plausible that women may start treatment later than men, which may contribute to poorer outcomes. ${ }^{5,6}$ Women may experience more lactic acidosis, drug toxicity and hypersensitivity reactions on antiretroviral
Correspondence: Curtis Cooper Associate Professor of Medicine, University of Ottawa, The Ottawa Hospital-General Campus, GI2-50I Smyth Rd, Ottawa, Ontario, Canada KIH 8 L6

Email ccooper@ottawahospital.on.ca 
therapy which may contribute to decreased adherence and treatment interruption. ${ }^{1,7}$ Some studies indicate that women have higher rates of clinical progression to acquired immunodeficiency syndrome (AIDS) and death while others have suggested the opposite..$^{1,2,8}$ Differential access to care for women between studies may be an important factor explaining these discrepancies. ${ }^{1,2,8}$ Many women with HIV report high rates of poverty, are often depended on for care of children or family members, and may show a reluctance to take medication in public due to denial or fear of disclosure which may lead to compromised self-care, access to care, and response to treatment. ${ }^{4,9}$ Depression disproportionally affects women and may contribute to worse outcomes, as depressive symptoms in women are associated with hastened HIV progression. ${ }^{10}$

Hepatitis C virus (HCV) coinfection in HIV is common and contributes to increased morbidity and mortality. ${ }^{11}$ Many of the barriers to HIV care access and treatment listed above are more frequent and more severe in those with HIV-HCV coinfection. ${ }^{12,13}$ Furthermore, HCV itself may negatively influence the natural history of HIV disease and therapeutic response to HAART. ${ }^{11}$ The interaction between HIV, HCV, and sex is an area that remains largely unexplored. Well-established gender differences between sexes in HIV-HCV coinfected women include greater likelihood of spontaneous clearance of acute HCV infection and higher symptom burden. ${ }^{14,15}$ There is no literature evaluating differences in HIV-related outcomes in the HIV-HCV coinfected population. To this end, we evaluated the influence of biological sex on HIV antiretroviral clinical, immunological, and virological outcome in a well-described HIV-HCV coinfected population followed in a tertiary care-based, specialized immunodeficiency clinic. Specific data on sex and HIV-HCV coinfection is important, as women are a rapidly growing segment of the overall HIV population. ${ }^{16,17}$

\section{Methods}

We assessed all HAART-treated HCV-HIV coinfected individuals followed at The Ottawa Hospital Immunodeficiency Clinic from January 1996 to June 2008. We identified patients in a prospective cohort of clinic attendees and supplemented this data by chart review. HIV RNA viral load, CD4 lymphocyte counts, liver enzymes and function tests, and HCV serology results were collected. Characteristics including age, history of excess alcohol, history of injection drug use (IDU), treatment start date, HAART composition, and pregnancy status were identified.
Primary outcome measures included duration of therapy before interruption or change, suppression of HIV RNA levels below detection within 6 and 12 months, mean CD4 counts after 6 months of HAART, and cumulative survival. The primary reason for treatment interruption was assessed. Categories included gastrointestinal side effects (eg, nausea, vomiting, abdominal discomfort, diarrhea), neuropsychiatric symptoms (eg, depression, headaches, difficulties with mentation, neuropathy), adherence difficulty, metabolic complications, liver toxicity, renal complications, musculoskeletal side effects, hematologic abnormalities, dermatologic complications, alcohol and/or substance abuse, pill count or dosing frequency issues, virologic failure, and death. Measures of adherence were based on self-report and clinician suspicions of nonadherence.

We assessed baseline characteristics using parametric testing ( $t$-tests and Chi-squared). Our primary outcome measures were analyzed using time to event analysis with a step-wise multivariable analysis. We consider a $P$-value of less than 0.05 as significant and all $P$-values are two-sided. Collection and analysis of this data was approved by the Ottawa Hospital Research Ethics Board.

\section{Results}

A total of 183 HIV-HCV coinfected HAART-treated patients followed from January 1996 to June 2008 were assessed (Table 1). Females made up $21 \%$ of the evaluated population. The majority were White males. A high level of excess alcohol use and history of IDU was noted in both

Table I Baseline characteristics of HIV-HCV coinfected patients

\begin{tabular}{lll}
\hline Characteristic & Male & Female \\
& $\mathbf{N}=144$ (79\%) & $\mathbf{N = 3 9}$ (2 I \%) \\
\hline Mean age [yrs (SD)] & $38.4(7.2)$ & $36.6(7.6)$ \\
Mean baseline HIV RNA Level & 96,433 & 72,922 \\
[copies/mL (SD)] & $(162,634)$ & $(113,227)$ \\
Mean baseline CD4 count & $290(226)$ & $375(387)$ \\
[cells/ $\mu$ L (SD)] & & \\
Baseline CD4 count & $37 \%$ & $42 \%$ \\
$<200$ cells/ $\mu$ L & & \\
Mean ALT [U/L (SD)] & $66(72)$ & $51(42)$ \\
History of excess alcohol & $37 \%$ & $36 \%$ \\
History IDU & $76 \%$ & $85 \%$ \\
White & $86 \%$ & $79 \%$ \\
Black & $8 \%$ & $13 \%$ \\
Asian & $<1 \%$ & $0 \%$ \\
Native & $5 \%$ & $8 \%$ \\
\hline
\end{tabular}

Abbreviations: ALT, alanine aminotransferase; $\mathrm{HCV}$, hepatitis $\mathrm{C}$ virus; $\mathrm{HIV}$, human immunodeficiency virus; IDU, injection drug use; SD, standard deviation. 
sexes. A trend toward lower baseline HIV RNA levels and higher CD4 counts was noted in women. Four women (10\%) initiated HAART specifically for pregnancy. There was no difference in baseline CD4 counts between these four patients and the other 35 females who started HAART. None of these four women or two others who became pregnancy while on HAART was exposed to efavirenz.

There were subtle differences in specific drugs chosen when assessed by sex (Table 2). A higher proportion of men initiated high-dose ritonavir and low-dose ritonavir-boosted containing therapy. More females initiated ritonavir-sparing (ie, nelfinavir, atazanavir) treatment. Women were less likely to initiate efavirenz which may reflect concerns related to teratogenicity risk in women of child-bearing age. Because the median start date for HAART was similar by sex (August 1999 for men; December 1998 for women), we are confident that these differences in antiretroviral composition by sex are not a consequence of a treatment era effect bias. Median duration of follow-up after the initiation of therapy was 4.7 years for men and 5.1 years for women $(P=0.55)$. The median duration on therapy was 4 months for women (range: 1 to 10 months) and 10 months for men (range: 4 to 24 months) $(P=0.01)$ (Figure 1). The duration on therapy did not differ for women started on HAART for pregnancy compared to women initiated on treatment for other indications (data not shown). At last assessment, only $9 \%$ of the men and $8 \%$ of the women were on the same treatment regimen as the one started revealing a high turnover rate of antiretrovirals. Twelve percent of women and $15 \%$ of men stopped antiretroviral therapy without resumption, $35 \%$ of women and $48 \%$ of men immediately changed to a second

Table 2 HAART composition for HIV-HCV patients starting treatment

\begin{tabular}{lll}
\hline & Male $(\mathbf{n}=1 \mathbf{4 4})$ & Female $(\mathbf{n}=\mathbf{3 9})$ \\
\hline Protease inhibitor-based HAART & $69 \%(\mathrm{n}=100)$ & $77 \%(\mathrm{n}=30)$ \\
Ritonavir & $70 \%$ & $47 \%$ \\
High-dose ritonavir & $39 \%$ & $30 \%$ \\
Saquinavir & $41 \%$ & $40 \%$ \\
Indinavir & $14 \%$ & $20 \%$ \\
Nelfinavir & $17 \%$ & $23 \%$ \\
Kaletra & $23 \%$ & $7 \%$ \\
Atazanavir & $0 \%$ & $10 \%$ \\
NNRTI-based HAART & $22 \%(n=31)$ & $18 \%(n=7)$ \\
Efavirenz & $77 \%$ & $71 \%$ \\
Nucleoside-sparing & $10 \%$ & $0 \%$ \\
\hline
\end{tabular}

Abbreviations: HAART, highly-active antiretroviral therapy; NNRTI, non-nucleoside reverse transcriptase inhibitors. line regimen without interruption, and $53 \%$ of women and $37 \%$ of men resumed therapy but only after an interruption in HAART $(P=0.24)$.

Mean CD4 counts increased to 413 cells/ $\mu \mathrm{L}$ in women and 400 cells $/ \mu \mathrm{L}$ in men after 6 months of HAART and did not differ by sex. The absolute increase in CD4 cells from baseline was greater in males (102 versus 11 cells $/ \mu \mathrm{L}$; $P=0.04)$. The proportion achieving virological suppression below the lower limit of detection were similar at 6 months (women: 77\%, men 74\%; $P=0.73$ ) and 12 months (women: $82 \%$, men: $81 \% ; P=0.87$ ) of therapy in those remaining on HAART at these time points.

Both women $(25 \%)$ and men $(19 \%)(P=0.42)$ cited gastrointestinal complications as the primary reason for HAART interruption (Table 3). Adherence difficulties were a common reason for discontinuation in women $(22 \%)$ and men $(15 \%)$ $(P=0.31)$. Women were more likely to stop treatment due to neuropsychiatric issues $(P=0.003)$. The use of efavirenz was similar in those interrupting therapy for neuropsychiatric complications $(23 \%)$ and those not $(15 \% ; P=0.46)$. Two of six men and one of seven women on efavirenz who stopped treatment for neuropsychiatric reasons did so specifically due to efavirenz-attributed side effects. Men were more likely to be lost to follow-up $(P=0.08)$. Liver complications were an infrequent cause for HAART change or interruption, irrespective of sex. Seven males $(5 \%)$ and no females discontinued therapy for liver-specific complications.

Mortality analysis revealed an association between female sex and reduced cumulative survival (odds ratio $[\mathrm{OR}]=4.46,95 \%$ confidence intervals $[\mathrm{CI}]: 1.8-11.0$; $P=0.001$ ) (Figure 2). When controlled for IDU, race, and baseline CD4, this association remained significant. Twentythree percent of women and $7 \%$ of men died during the period of evaluation $(P=0.003)$. Sixty percent of deaths were end-stage liver disease-related and did not differ by sex.

\section{Discussion}

There is evidence that biological sex may influence HIV progression and treatment response. ${ }^{1,7}$ These differences are of ever-increasing importance given that women comprise a rapidly growing segment of the HIV population. ${ }^{16,17}$ Little is known as to how HCV coinfection influences the interaction between HIV and biological sex. Our findings indicate that HIV-HCV coinfected women are more likely to interrupt or change treatment before men, differ in reasons for therapy interruption, and may have a lower cumulative survival. These differences are not explained by the subtle baseline differences in characteristics. 


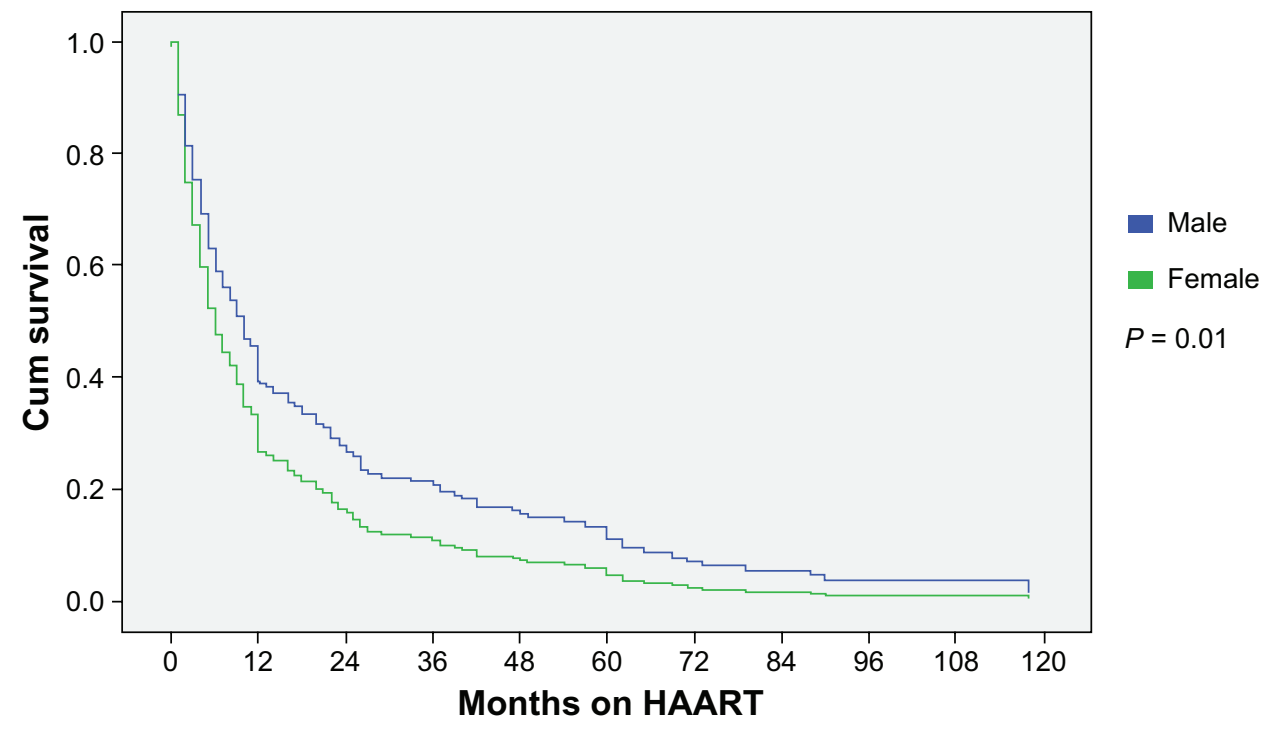

Figure I Months on HAART by sex prior to interruption or treatment change. Abbreviation: HAART, highly-active antiretroviral therapy.

When comparing duration on HAART prior to interruption or change in antiretroviral(s), differences between men and women were most apparent during the first 12 months of therapy (Figure 1). Thereafter, the rates were similar. The reason for differences in duration of therapy before interruption or change is unclear but likely multifactorial. Similar findings have been observed in HIV mono-infected populations. ${ }^{1}$ The proportion of men and women on the same HAART regimen after one year was low and did not differ by sex. Women more typically interrupted HAART with a break prior to resumption of therapy whereas men where more likely to immediately switch to an alternate regimen. The reason for this is unclear. However, post-partum discontinuation of therapy did not explain this observation.

It is plausible that pregnancy may influence the timing of HAART initiation and therapeutic outcomes in HIV. Pregnancy may affect pharmacokinetics that could influence side effects. ${ }^{18-20}$ Pregnancy may contribute to greater drug intolerance in female recipients and higher rates of treatment discontinuation as following delivery HAART may be

Table 3 Primary reasons for therapy interruption by sex

\begin{tabular}{llll}
\hline & Female & Male & $\boldsymbol{P}$ \\
\hline Gastrointestinal complaints & $25 \%$ & $19 \%$ & 0.42 \\
Adherence & $22 \%$ & $15 \%$ & 0.31 \\
Mental health & $19 \%$ & $5 \%$ & 0.003 \\
Lost to follow-up & $3 \%$ & $13 \%$ & 0.08 \\
Substance use & $5 \%$ & $6 \%$ & 0.79 \\
Liver & $0 \%$ & $5 \%$ & 0.06 \\
\hline
\end{tabular}

stopped depending on patient and physician preferences towards remaining on treatment (dependent on disease stage). Consistent with other studies, we did not identify a pregnancy effect on HIV disease progression or outcome. ${ }^{21,22}$ However, the number of pregnancies in our cohort was low.

In our study, virological and immunological outcomes did not differ by sex in those remaining on therapy. It is noteworthy that the baseline CD4 count at treatment initiation was higher in women. This difference was not explained by pregnant females with high CD4 counts initiating HAART to prevent maternal to fetal HIV transmission. It is plausible that more women initiated HAART earlier in the HAART era when higher CD4 counts triggered the introduction of therapy. This was not the case in our cohort as the average date of treatment initiation was similar by sex. We suspect that better compliance with scheduled clinic visits in women may have contributed to HAART-initiation at higher CD4 counts. There are discrepancies between other studies with some reporting higher, ${ }^{23}$ lower $^{2}$ or similar ${ }^{1,724,25}$ baseline CD4 counts by sex. This may reflect the influence of the above mentioned confounders.

Consistent with other work, the primary reason for therapy interruption differed by sex in our analysis. ${ }^{26}$ Treatment adherence in our female patients was not a significant factor leading to treatment interruption or change. This finding is consistent with some, but not all analyses. ${ }^{24,27}$ It is unclear why so many men where lost to follow-up and to what effect this had on the outcomes in the study. ${ }^{3}$ Previous research by our group indicated that our HCV-HIV coinfected population faces multiple barriers to treatment including substance 


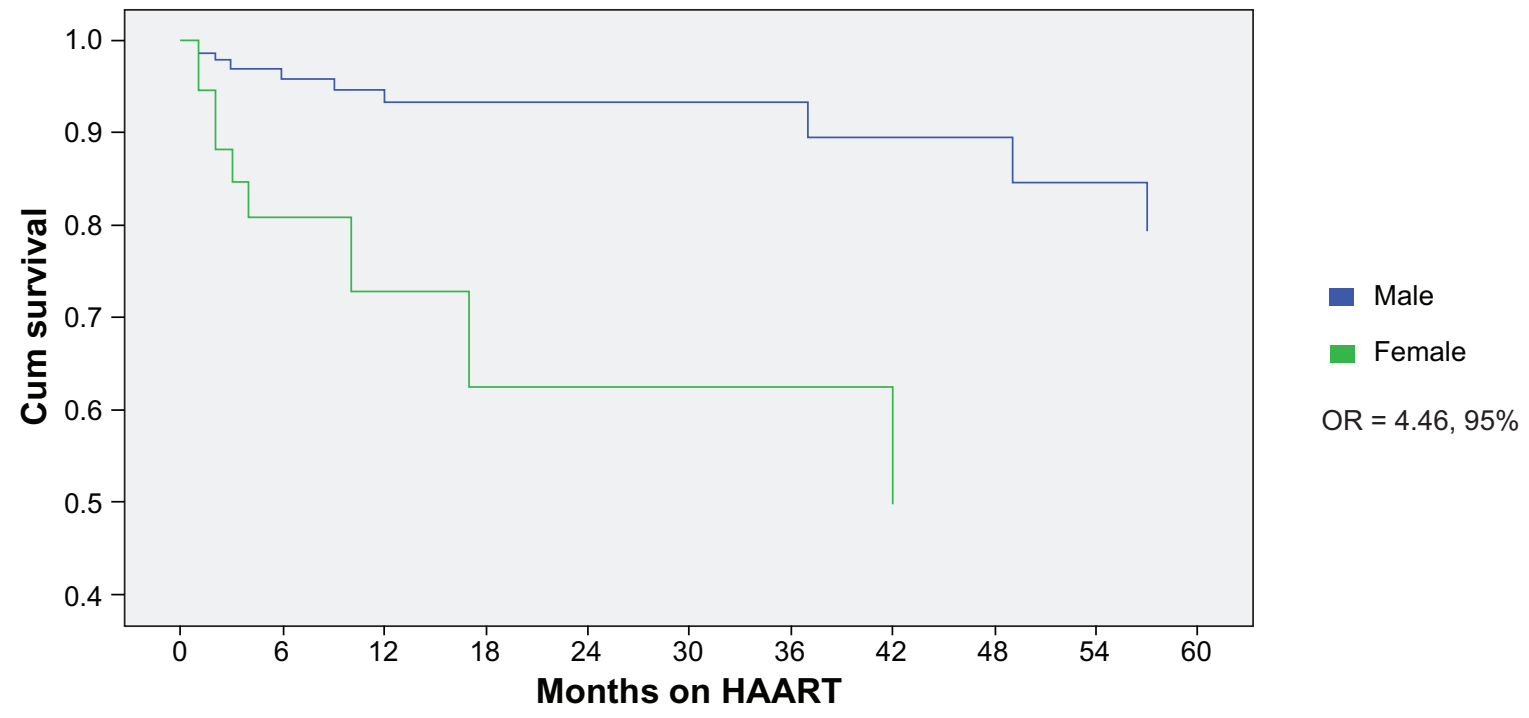

Figure 2 Comparison of cumulative survival by sex.

Abbreviations: $\mathrm{Cl}$, confidence intervals; $\mathrm{HAART}$, highly-active antiretroviral therapy; OR, odds ratio.

abuse, psychiatric illness, anxiety related to workup or treatment, and poor social circumstances which contribute to suboptimal follow-up. ${ }^{4}$

Neuropsychiatric issues were identified as an important reason for therapy interruption in females. Depression and other mental health issues are common in HIV-HCV coinfection. ${ }^{28-30}$ In $\mathrm{HCV}$ populations, current levels of psychiatric disorders range from $31 \%$ to $58 \%$ with lifetime rates as high as $95 \% .{ }^{5}$ This may be higher in women compared to men. ${ }^{10}$ In HIV populations, psychiatric disorders are also common with up to $36 \%$ of individuals suffering from major depression, a rate that is three times higher than non-HIV infected individuals. ${ }^{31,32}$ Several studies suggest mental illness is more prevalent and more severe in the HIV-HCV coinfection. ${ }^{31-34}$ Studies evaluating HIV mono-infected patients suggest that much of the variation in HIV progression is related to depression, stressful life events, and trauma. ${ }^{32}$ In our analysis women were more likely to have a psychiatric illness and for this to affect their treatment. Mental health issues may also explain why women in our study had a decreased cumulative survival. Despite the well-established influence on mental health, the use of efavirenz did not appear to influence the likelihood of interrupting HAART in our cohort. ${ }^{35}$ This data highlight the importance of thoroughly evaluating and treating mental health issues, especially in women, as such interventions may significantly improve outcomes in HIV-HCV coinfection.

Patients with HIV-HCV coinfection have higher mortality rates. ${ }^{11}$ Little is known about gender differences among coinfected populations. Our results suggest that in the HIV-HCV coinfected population, mortality in women may be higher despite similar viral suppression and CD4 counts, and when controlling for IDU history, race, and baseline CD4 levels. Recent data in HIV mono-infected individuals reveals that women generally live longer with HIV, which contradicts our findings and may indicate that the negative interaction between these two viruses is particularly harmful in women. ${ }^{6}$ The primary cause for death was related to end-stage liver disease. HCV-related liver disease does progress more rapidly in women, which partially explains our results..$^{36}$ This suggests that HIV-HCV coinfected women should be aggressively evaluated and treated for HCV. Mental health issues in women may have contributed to our findings. Others have reported that HIV-positive women with chronic depressive symptoms are two times more likely to die and show greater declines in CD4 counts. ${ }^{10}$ We do not believe that the differential mortality rates observed in our analysis are a consequence of an era effect as the average date of initiation of HAART and the median duration of follow-up was similar by sex. We acknowledge that the high number of loss to follow-up in males may have skewed the data as the true outcomes of these patients, including the possibility of death, is unknown. Furthermore, it is difficult to separate biological differences from confounding socioeconomic and mental health factors which are well-established to influence adherence, morbidity, and survival. ${ }^{12,13}$

There are several limitations to the current study. Most patients were White. It is unclear whether race directly influences the natural history of HIV-HCV coinfected individuals. ${ }^{22}$ The patients evaluated in this study were 
managed at a tertiary center offering specialized HIV care. Due to referral bias, a broader population of HIV-HCV coinfected individuals that are not referred or who have yet to seek care may have been missed. As a retrospective evaluation, reporting bias and missing data may have influenced our findings. Nonetheless, our analysis convincingly indicates that biological sex is not associated with differences in virological and immunological outcome in HIV-HCV coinfection initiating HAART. However, reasons for treatment interruption and mortality itself may differ by sex. These findings are of value in guiding health care providers as they plan and manage gender-specific treatment in HIV-HCV coinfection.

\section{Disclosures}

The authors report no conflict of interest in this work.

\section{References}

1. Collazos J, Asensi V, Carton JA. Sex differences in the clinical, immunological and virological parameters of HIV-infected patients treated with HAART. AIDS. 2007;21(7):835-843.

2. Cunningham WE, Markson LE, Andersen RM, et al. Prevalence and predictors of highly active antiretroviral therapy use in patients with HIV infection in the United States. HCSUS Consortium. HIV Cost and Services Utilization. J Acquir Immune Defic Syndr. 2000;25(2):115-123.

3. Furler MD, Einarson TR, Walmsley S, Millson M, Bendayan R. Longitudinal trends in antiretroviral use in a cohort of men and women in Ontario, Canada. AIDS Patient Care STDS. 2006;20(4): 245-257.

4. Gebo KA, Fleishman JA, Conviser R, et al. Racial and gender disparities in receipt of highly active antiretroviral therapy persist in a multistate sample of HIV patients in 2001. J Acquir Immune Defic Syndr. 2005;38(1):96-103.

5. Gandhi M, Bacchetti P, Miotti P, Quinn TC, Veronese F, Greenblatt RM. Does patient sex affect human immunodeficiency virus levels? Clin Infect Dis. 2002;35(3):313-322.

6. Farzadegan H, Hoover DR, Astemborski J, et al. Sex differences in HIV-1 viral load and progression to AIDS. Lancet. 1998;352(9139): $1510-1514$.

7. Boulassel MR, Morales R, Murphy T, Lalonde RG, Klein MB. Gender and long-term metabolic toxicities from antiretroviral therapy in HIV-1 infected persons. J Med Virol. 2006;78(9):1158-1163.

8. Hewitt RG, Parsa N, Gugino L. Women's health. The role of gender in HIV progression. AIDS Read. 2001;11(1):29-33.

9. Zierler S, Krieger N. Reframing women's risk: social inequalities and HIV infection. Annu Rev Public Health. 1997;18:401-436.

10. Ickovics JR, Hamburger ME, Vlahov D, et al. HIV Epidemiology Research Study Group. Mortality, CD4 cell count decline, and depressive symptoms among HIV-seropositive women: longitudinal analysis from the HIV Epidemiology Research Study. JAMA. 2001;285(11):1466-1474.

11. Greub G, Ledergerber B, Battegay M, et al. Clinical progression, survival, and immune recovery during antiretroviral therapy in patients with HIV-1 and hepatitis C virus coinfection: the Swiss HIV Cohort Study. Lancet. 2000;356(9244):1800-1805.

12. Falusi OM, Pulvirenti J, Sarazine J, Shastri P, Gail C, Glowacki R. HIV-infected inpatients in the HAART era: how do hepatitis C virus coinfected patients differ? AIDS Patient Care STDS. 2003;17(1): $13-16$.
13. Wood E, Kerr T, Tyndall MW, Montaner JS. A review of barriers and facilitators of HIV treatment among injection drug users. AIDS. 2008;22(11):1247-1256.

14. Antiretroviral Therapy Cohort Collaboration. Life expectancy of individuals on combination antiretroviral therapy in high-income countries: a collaborative analysis of 14 cohort studies. Lancet. 2008;372(9635):293-299.

15. Grebely J, Raffa JD, Lai C, Krajden M, Conway B, Tyndall MW. Factors associated with spontaneous clearance of hepatitis $\mathrm{C}$ virus among illicit drug users. Can J Gastroenterol. 2007;21(7):447-451.

16. Burruano L, Kruglov Y. HIV/AIDS epidemic in Eastern Europe: recent developments in the Russian Federation and Ukraine among women. Gend Med. 2009;6(1):277-289.

17. Farr SL, Kraft JM, Warner L, Anderson JE, Jamieson DJ. The integration of STD/HIV services with contraceptive services for young women in the United States. Am J Obstet Gynecol. 2009;201(2):142, e1-e8.

18. Bardeguez AD, Lindsey JC, Shannon M, et al. PACTG 1025 Protocol Team. Adherence to antiretrovirals among US women during and after pregnancy. J Acquir Immune Defic Syndr. 2008;48(4):408-417.

19. Clark R. Considerations for the antiretroviral management of women in 2008. Womens Health (Lond Engl). 2008;4(5):465-477.

20. Stek AM. Antiretroviral medications during pregnancy for therapy or prophylaxis. Curr HIV/AIDS Rep. 2009;6(2):68-76.

21. Ahdieh L. Pregnancy and infection with human immunodeficiency virus. Clin Obstet Gynecol. 2001;44(2):154-166.

22. Hershow RC, O'Driscoll PT, Handelsman E, et al. Hepatitis C virus coinfection and HIV load, CD4+ cell percentage, and clinical progression to AIDS or death among HIV-infected women: Women and Infants Transmission Study. Clin Infect Dis. 2005;40(6): 859-867.

23. Perez-Hoyos S, Rodríguez-Arenas MA, García de la Hera M, et al. Progression to AIDS and death and response to HAART in men and women from a multicenter hospital-based cohort. $J$ Womens Health (Larchmt). 2007;16(7):1052-1061.

24. Nicastri E, Leone S, Angeletti C, et al. Sex issues in HIV-1-infected persons during highly active antiretroviral therapy: a systematic review. J Antimicrob Chemother. 2007;60(4):724-732.

25. Sterling TR, Vlahov D, Astemborski J, Hoover DR, Margolick JB, Quinn TC. Initial plasma HIV-1 RNA levels and progression to AIDS in women and men. $N$ Engl J Med. 2001;344(10):720-725.

26. Kumarasamy N, Venkatesh KK, Cecelia AJ, et al. Gender-based differences in treatment and outcome among HIV patients in South India. J Womens Health (Larchmt). 2008;17(9):1471-1475.

27. Kuyper LM, Wood E, Montaner JS, Yip B, O'Connell JM, Hogg RS. Gender differences in HIV-1 RNA rebound attributed to incomplete antiretroviral adherence among HIV-Infected patients in a population-based cohort. J Acquir Immune Defic Syndr. 2004;37(4):1470-1476.

28. McLaren M, Garber G, Cooper C. Barriers to hepatitis C virus treatment in a Canadian HIV-hepatitis $\mathrm{C}$ virus coinfection tertiary care clinic. Can J Gastroenterol. 2008;22(2):133-137.

29. Restrepo A, Johnson TC, Widjaja D, et al. The rate of treatment of chronic hepatitis $\mathrm{C}$ in patients co-infected with HIV in an urban medical centre. J Viral Hepat. 2005;12(1):86-90.

30. Righi E, Beltrame A, Bassetti M, et al. Therapeutical aspects and outcome of HIV/HCV coinfected patients treated with pegylated interferon plus ribavirin in an Italian cohort. Infection. 2008;36(4): 358-361.

31. Douaihy A, Hilsabeck RC, Azzam P, Jain A, Daley DC. Neuropsychiatric aspects of coinfection with HIV and hepatitis C virus. AIDS Read. 2008;18(8):425-432, 438-439.

32. Leserman J. Role of depression, stress, and trauma in HIV disease progression. Psychosom Med. 2008;70(5):539-545.

33. Butt AA, Khan UA, Skanderson M. Comorbidities and their impact on mortality in HCV and HCV-HIV-coinfected persons on dialysis. J Clin Gastroenterol. 2008;42(9):1054-1059. 
34. Siika AM, Ayuo PO, Sidle MJ, Wools-Kaloustian K, Kimaiyo SN, Tierney WM. Admission characteristics, diagnoses and outcomes of HIV-infected patients registered in an ambulatory HIV-care programme in western Kenya. East Afr Med J. 2008;85(11):523-528.

35. Quereda C, Corral I, Moreno A, et al. Effect of treatment with efavirenz on neuropsychiatric adverse events of interferon in HIV/HCV-coinfected patients. J Acquir Immune Defic Syndr. 2008;49(1):61-63.
36. Rodríguez-Torres M, Ríos-Bedoya CF, Rodríguez-Orengo J, et al Progression to cirrhosis in Latinos with chronic hepatitis $\mathrm{C}$ : differences in Puerto Ricans with and without human immunodeficiency virus coinfection and along gender. J Clin Gastroenterol. 2006;40(4): $358-366$.

\section{Publish your work in this journal}

Patient Preference and Adherence is an international, peer-reviewed, open access journal that focusing on the growing importance of patient preference and adherence throughout the therapeutic continuum. Patient satisfaction, acceptability, quality of life, compliance, persistence and their role in developing new therapeutic modalities and compounds to optimize clinical outcomes for existing disease states are major areas of interest. This journal has been accepted for indexing on PubMed Central The manuscript management system is completely online and includes a very quick and fair peer-review system. Visit http://www.dovepress.com/ testimonials.php to read real quotes from published authors.

Submit your manuscript here: http://www.dovepress.com/patient-preference-and-adherence-journal 